\title{
Computed Tomography Angiography Evaluation of Peripheral Arterial Disease and Correlation with Colour Doppler
}

\author{
M. Balaji Vara Prasad ${ }^{\oplus 1}$, Srinadh Boppana ${ }^{\odot 2}$, Mounika Reddy ${ }^{\circledR 2}$, Nainika Juvvadi ${ }^{3}$, Rajeshwari ${ }^{\circ}$, Radhika ${ }^{\circ}$ \\ ${ }^{1}$ Associate Professor, KAMINENI institute of medical sciences and Research Centre, Hyderabad, Telangana, India, ${ }^{2}$ Senior Consultant, KAMSRC, Hyderabad, Telangana, \\ India, ${ }^{3}$ Resident, KAMSRC, Hyderabad, Telangana, India.
}

\section{Abstract}

Background: Peripheral Arterial Disease Is a Major Problem Among the Population of Those 55 Years and Older. MDCT is a Non-Invasive Method of Visualization of Vascular System. It Affords More Widespread Vascular Screening, Allowing Diagnosis to be Made at an Early Stage. The objective is to Assess the Role of Multidetector Computed Tomography Angiography in Peripheral Arterial Disease of Lower Extremities. Subjects \& Methods: A Prospective Study of 35 Patients with Signs and Symptoms of PVD, Were Subjected to Colour Doppler Ultrasonography and MDCT Examination Between a Period of $1^{s t}$ August 2018 to $31^{\text {st }}$ May 2019. Results: Our Study Shows a Very Good Sensitivity, Specificity, PPV, NPV and Accuracy of Doppler in Assessing the Soft Plaque (82\%, 100\%, 100\%, 97\%, 97\% Respectively) \& in Evaluation of Stenosis Like $<50 \%$ Stenosis $(100 \%, 99 \%, 85 \%, 100 \%$ \& 99\% Respectively), 50-99\% Stenosis (100\%, 99\%, 96\%. 100\% \& 99.8\% Respectively), \& Occlusion (84\%, 100\%. 100\%, 98\% \& 98\% Respectively). The Only Parameter Which Showed Poor Result Was in Evaluation of Calcified Plaque. Conclusion: Our Study Shows that Both Colour Doppler \& MDCT Can Be Complimentary in Lower Limb Arterial Disease Evaluation.

Keywords: Peripheral Arterial Disease, Plaque, Stenosis, Doppler, Multi-Detector Computed Tomography.

Corresponding Author: M. Balaji Vara Prasad, Associate Professor, KAMINENI institute of medical sciences and Research Centre, Hyderabad, Telangana, India.

E-mail: balajidrmallula@yahoo.com

Received: 05 October 2020

\section{Introduction}

Peripheral arterial disease (PAD) refers to atherosclerotic disease leads to development of plaques in the distal arteries, causing stenosis with compromised blood flow. It is more common in lower limbs than upper limbs. ${ }^{[1]}$ Atherosclerosis of arteries distal to the aortic bifurcation called lowerextremity PAD. It is major problematic disease after 55 years. Clinical features appears first as intermittent claudication, then it progresses to critical limb ischemia leads to rest pain and necrosis of tissues. ${ }^{[1]}$ In south indian population it's prevalence rate is $3.2 \%{ }^{[2,3]}$

In evaluation of peripheral vascular disease digital subtraction angiography (DSA), acts as a 'gold standard' method. However recent advances in color Doppler, multi-detector computed tomography (MDCT) and contrast-enhanced magnetic resonance angiography (MRA) challenges the role of DSA. ${ }^{[4]}$ Colour Doppler ultrasonography is a non-invasive method with good specificity and sensitivity. Doppler provides both anatomical and functional information, but it is not ideal for
Accepted: 07 December 2020

Published: 30 December 2020

planning treatment. MDCT is a minimally invasive method of visualization of vascular system. It affords more widespread vascular screening, allowing diagnosis to be made at an early stage. ${ }^{[5]}$

\section{Aim}

To assess the role of multidetector computed tomography angiography in lower limb peripheral arterial disease.

\section{Objectives}

To Evaluate the Lower Limb Arterial Diseases severity.

To Compare the Doppler Ultrasonography Findings with MDCT Angiography in lower limb Peripheral Arterial Disease.

\section{Subjects and Methods}

This is a Prospective Study done on 35 Patients with Signs and Symptoms of Pad, during the Period of $1^{\text {st }}$ August 2018 to $31^{\text {st }}$ May 2019. 


\section{Inclusion Criteria}

- All Peripheral Arterial Diseased syptomatic patients.

- Burger's Disease.

- Aorto Arteritis with Secondary Involvement of Peripheral Vessels

- Secondary Involvement of Arteries Due to Tumour.

- Post Traumatic Injuries to Peripheral Vessels.

\section{Exclusion Criteria}

- Patients with Severe Renal Insufficiency, EGFR $<30$

- Patients with H/O Adverse Reactions to Contrast Agents.

- Follow-up Study of PVD with Grafts.

\section{Results}

\section{Patients}

Total of thirty-five patients with peripheral arterial disease were examined. Of the thirty-five patients, thirty-two (91.4\%) were male patients and three $(8.6 \%)$ were female patients. Majority of subjects $42.9 \%$ were in the age group 46-60yrs followed by $25.7 \%$ were $>60 y r s, 17.1 \%$ were in $31-45 y$ rs age group and $14.3 \%$ were in 16-30yrs age group.

Out of 35 patients, $17(48.6 \%)$ patients had history of diabetes, $10(28.6 \%)$ patients had history smoking/tobacco chewing, $4(11.4 \%)$ patients had trauma, $4(11.4 \%)$ patients had no significant history. Seventy limbs of 35 patients were studied. Some 630 arterial segments in 70 limbs (210 iliac segments, 140 femoral segments, 70 popliteal segments, 210 (Tibioperoneal segments) were examined.

\section{Plaque Evaluation}

\section{Calcified Plaque}

In the iliac segment, out of 210 segments, calcified plaque was found in only 28 segments on Doppler whereas MDCT could detect 47 segments with calcified plaque. In the femoral segment out of 140 segments, calcified plaque was found in only 23 segments on Doppler whereas MDCT could detect 26 segments with calcified plaque. In the popliteal segment, out of 70 segments, Doppler detected only 16 segments and MDCT detected 18 segments. In the Tibio peroneal segments out of 210 segments, Doppler detected 27 segments, MDCT detected 35 segments with calcified plaque. Doppler was poor in detecting calcified plaque in all the three segments, when compared to MDCT.

\section{Soft Plaque}

In iliac segment, out of 210 segments, soft plaque was found in 16 segments on Doppler whereas MDCT could detect
19 segments. Doppler missed 3 segments of external iliac artery with soft plaque and Doppler detected soft plaque in one segment of internal iliac artery which was not found on MDCT. In femoral segments, out of 140 segments, soft plaque was found in 24 segments on Doppler whereas MDCT could detect 28 segments having soft plaque. Doppler missed 2 segments of common femoral artery \& 2 segments of superficial femoral artery. In popliteal segment, out of 70 segments, Doppler could detect 14 segments \& MDCT could detect 16 segments with soft plaque. In Tibio - peroneal segment, out 210 segments, Doppler could detect 20 segments \& MDCT could detect 27 segments with soft plaque. Doppler missed 4 segments of peroneal artery \& 5 segments of anterior Tibial artery. Doppler detected soft plaque in 2 segments of posterior Tibial artery which was not found on MDCT.

\section{Evaluation Of Stenosis}

\section{Less Than $50 \%$ Stenosis}

In the iliac segments, out of 210 segments, Doppler detected 1 segments \& MDCT could detect 1 segment with less than $50 \%$ stenosis. In the femoral segments, out of 140 segments, Doppler could detect 2 segments \& MDCT could detect 3 segments with less than $50 \%$ stenosis. In the popliteal segments, both Doppler and MDCT could not detect any lesion with less than $50 \%$ stenosis. In the Tibio-peroneal segments, out of 210 segments, Doppler could detect 4 segments \& MDCT could detect 2 segments with less than 50\% stenosis. Doppler detected 3 segments of posterior Tibial artery with $<$ $50 \%$ stenosis but MDCT showed only calcified plaque with no stenosis, but Doppler missed 1 segment of anterior Tibial artery with $<50 \%$ stenosis.

\section{$50-99 \%$ Stenosis}

In the iliac segments, out of 210 segments, doppler detected 4 segments \& MDCT could detect 5 segments with $50-99 \%$ stenosis. Doppler missed 1 segment of common iliac \& 2 segments of internal iliac arteries. In the femoral segments, out of 140 segments, doppler could detect 5 segments and mdct could detect 3 segments with 50-99\% stenosis. Doppler detected 2 segments of superficial femoral artery with 50 $99 \%$ stenosis, whereas MDCT did not show the findings. In popliteal segment, out of 70 segments, doppler could detect 3 segments and mdct could detect 4 segments with $50-99 \%$ stenosis. In the tibio peroneal segments, out of 210 segments, doppler could detect 21 segments and MDCT could detect 20 segments with 50-99\% stenosis. Doppler detected 1 segment of anterior tibial artery, but MDCT showed good opacification due to collateral circulation.

\section{Occlusion}

In the iliac segments, out of 210 segments, doppler detected 11 segments \& MDCT also detected 12 segments with complete 
occlusion. MDCT detected 2 segments of external iliac artery which is not picked up in doppler. In the femoral segments, out of 140 segments, doppler could detect 18 segments \& MDCT could detect 21 segments with complete occlusion. 2 segments of common femoral artery \& 1 segment of superficial artery showed patchy flow whereas MDCT showed complete occlusion.in popliteal segment, out of 70 segments, doppler could detect 12 segments and MDCT could detect 13 segments with complete occlusion. In the tibio-peroneal segments, out of 210 segments, doppler could detect 17 segments \& MDCT could detect 23 segments with complete occlusion. 4 segments of posterior tibial artery showed patchy flow on doppler whereas MDCT showed complete occlusion.

\section{Discussion}

The aim of our study is to evaluate lower limb arterial disease with new imaging modality such as MDCT and correlating the findings with that of colour doppler.

\section{Age Distribution}

In our study majority of subjects $42.9 \%$ were in the age group $46-60 y$ rs followed by $25.7 \%$ were $>60 y r s, 17.1 \%$ were in 31 $45 y$ rs age group and $14.3 \%$ were in $16-30 y$ rs age group. This is in comparison with study done by Parikh et al, 2017 i.e majority of patients age range was of 33-75years. ${ }^{[6]}$ This is in comparison with study done by Premkumarchidambaram et al, i.e fifty patients with median age- 47.5 years. ${ }^{[7]}$ in other study by Rahul J Shirol et al, 2015 i.e 10 out of 30 patients, 10 belonged to 61-70 yrs. Age group (33.3\%), 9 patients - 51-60 yrs. $(30 \%), 2$ - less than 40 yrs. $(13.3 \%) .{ }^{[8]}$

\section{Sex Distribution}

In our study majority of subjects $32(91.4 \%)$ were males and only $3(8.6 \%)$ were females. This is in comparison with studies done by Hasa et al, 2016, 135 patients $(85 \%)$ were males and 25 patients were females ( $15 \%){ }^{[9]}$ Peedikayil et al, 2016, majority of the cases are males. ${ }^{[10]}$

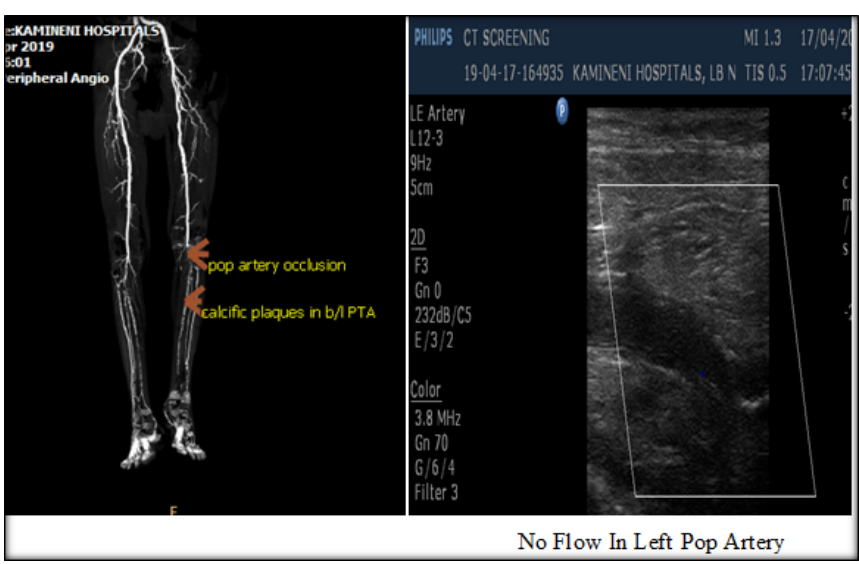

Figure 1: (A) 48 Years Male Patient Came with $\mathrm{C} / \mathrm{O}$ Bilateral Lower Limb Claudication Pain. MDCT Shows Occlusion of Left Distal Popliteal Artery and Bilateral Posterior Tibial Arteries. Doppler Shows Occlusion of Distal Popliteal Artery With Patchy And Dampened Flow In Bilateral Pta.)

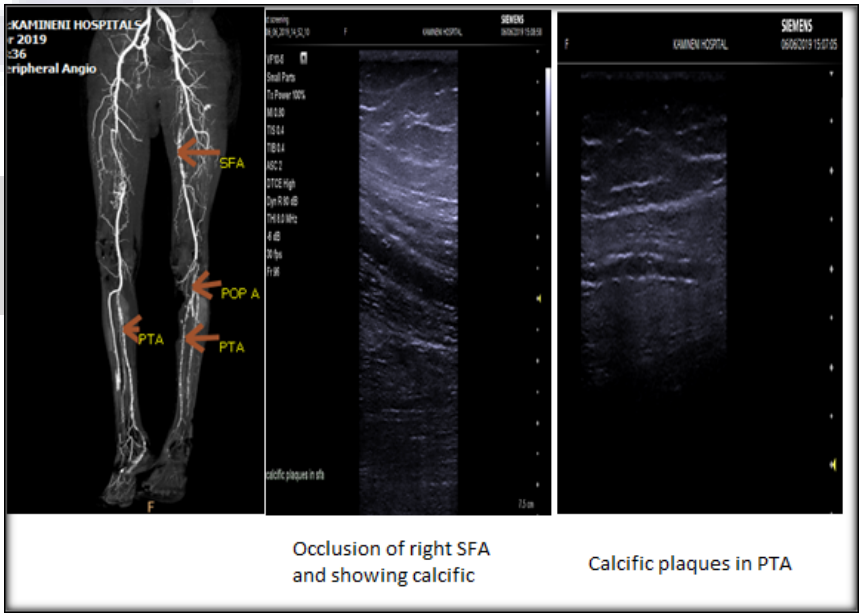

Figure 2: (A 65 Years Old Male Patient with H/O Smoking and Diabetes, C/O Claudication and Rest Pain in Bilateral Lower Limbs. MDCT Showed Occlusion Of Entire Right SFA, Diffuse Disease With Dense Calcified Plaques In B/L ATA, PTA Causing Occlusion And In Left SFA Causing Luminal Narrowing. Doppler Showed Right Sfa Occlusion And Calcified Plaques In B/L ATA, PTA.) 

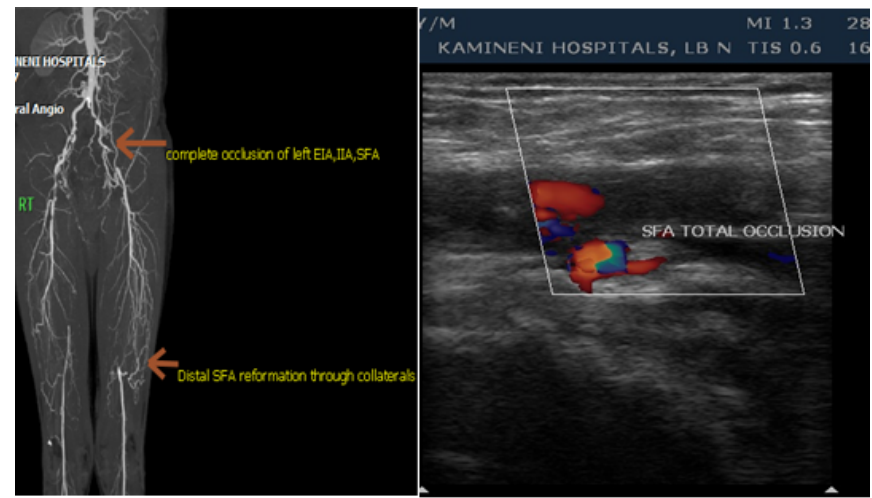

Figure 3: (A 50 Years Old Male Patient,H/O Diabetes, C/O Pain In Both Legs Severe On Left Side. MDCT Shows Complete Occlusion Of Left EIA, LIA, CFA And Bilateral SFA With Distal Reformation Through Multiple Collaterals.)

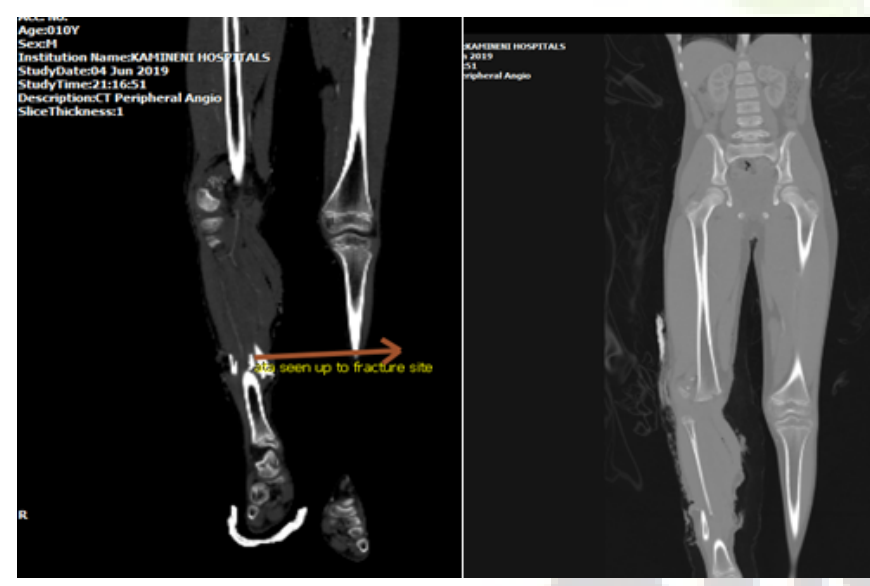

Figure 4: (A 10-Year-Old Male Came with H/O Fall for Assessment Vascular Injury. MDCTShows Occlusion of ATA Beyond the Fracture. Same Has Been Reported in Colour Doppler)

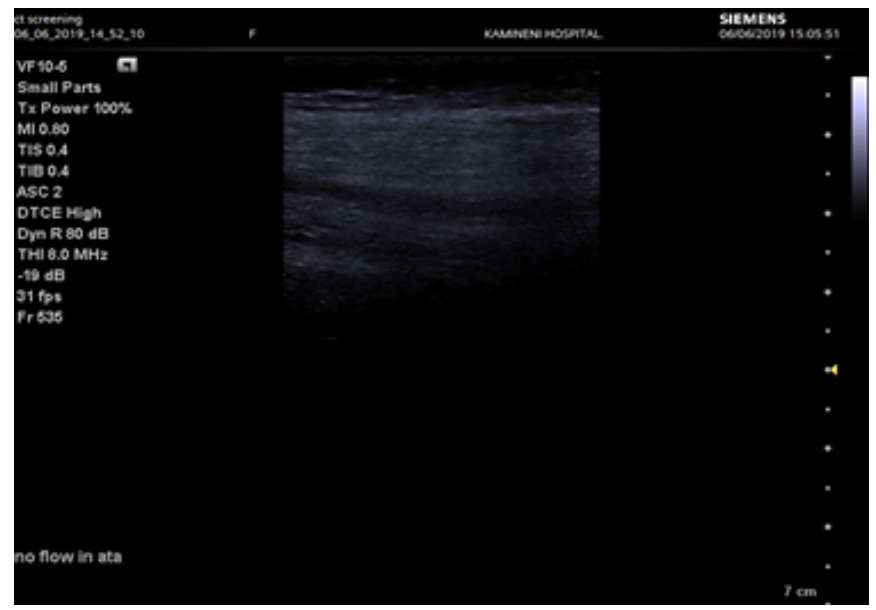

\section{Imaging}

\section{Evaluation of Calcified Plaque}

Sathyabhuwan et al, is a prospective study conducted on 50 patients. Calcific plaques are better detected on MDCT than colour doppler. ${ }^{[11]}$ Hideki ota et al, in a prospective study assessed the extent of calcification on MDCT angiography and found that MDCT showed 100\% sensitivity, specificity $\&$ accuracy in detecting mural calcification causing $<50 \%$ stenosis. ${ }^{[12]}$ In our study MDCT was able to detect calcified plaque in more arterial segments than colour doppler. Especially in iliac group, MDCT could detect 47 segments in comparison to 28 segments detected on doppler and in tibioperoneal segments, out of 210 segments, doppler detected 27 segments \& MDCT detected 35 segments with calcified plaque.in our study, the overall sensitivity, specificity, positive $\&$ negative predictive values and accuracy of doppler in detecting calcified plaque is $74 \%, 100 \%, 100 \%, 94 \%$ \& $94 \%$ respectively.

This-indicates that MDCT is more sensitive in detecting calcified plaque, when compared to doppler. This is because we carefully evaluated both axial \& reconstructed images for assessing calcified plaque. We were unable to detect calcified plaque on doppler especially in internal iliac artery where MDCT could detect 5 segments of internal iliac artery with calcified plaque. The low sensitivity of doppler in detecting calcified plaque is due to the presence of plaque in these segments with no significant stenosis and also may be due to subjective assessment of operator.

\section{Evaluation of Soft Plaque}

Duddalwar et al, study shows that characterization of atherosclerotic plaques and the presence or absence of calcification within it can be done by MDCT but not with DSA. ${ }^{[13]}$ In our study. MDCT was better in detecting soft plaques compared to doppler. In iliac \& popliteal segments doppler missed 3 segments, in femoral \&tibio-peroneal segments, doppler missed 4 and 7 segments with soft plaque, whereas MDCT showed soft plaque in all above segments. As stated in various studies, our study also showed that MDCT is better in characterization of plaque.

\section{$\underline{\text { Evaluation of Stenosis }}$}

\section{Less Than $\mathbf{5 0 \%}$ Stenosis}

Hideki Ota Et Al, Indicated That With Regard to The Detection of Segments That Had More Than Mild Stenosis, The Sensitivity, Specificity, And Accuracy of MDCT Angiography Were 99.2\%, 99.1\%, And 99.1\%, Respectively. ${ }^{[12]}$

In Our Study, The Overall Sensitivity, Specificity, PPV. NPV, Accuracy of Doppler Is 100\%, 99\%, 85\%, 100\% 
\& 99.8\% Respectively. This Indicates That Doppler Shows Excellent Sensitivity \&Specificity for Detecting Less Than $50 \%$ Stenosis, When Compared to MDCT.

\section{0 - 99\% Stenosis}

Albrecht et al, compared CT angiography performed on a 16MDCT scanner and DSA in patients with peripheral arterial disease. He found that MDCT had $93.3 \%$ sensitivity and $96.5 \%$ specificity for the detection of $(>50 \%)$ lesions. But with improved CT scanner technology \& increased slice scanner technology and increased slice scanner will further increase the above findings. ${ }^{[14]}$

The overall sensitivity is $100 \%$, specificity is $99.8 \%$, positive $\&$ negative predictive values are $96.9 \%$ and 100 and accuracy is $99.8 \%$ in detecting $50-99 \%$ stenosis. These results are comparable to the results of the previous studies done.

\section{Occlusion}

Thomas Schertler et al, studied the analysis of section width in arterial occlusive disease of lower limb with sixteen detector CT and found that with thinnest possible section the sensitivity, specificity and accuracy was $95 \%, 95 \% \& 96 \%$ respectively. ${ }^{[15]}$ The overall sensitivity, specificity, positive \& negative predictive values and accuracy of doppler in detecting $50-99 \%$ stenosis is $84 \%, 100 \%, 100 \%, 98.1 \% \& 98.3 \%$ respectively, Kayhan et al, in their study concluded that MDCTA could be used as a screening tool in patients with mild lower limb pad as it is a more accurate method compared to DUS. ${ }^{[16]}$ In our study we also found that colour doppler ultrasound was significantly lower statistically than MDCTA. Romano et, al concluded that in comparision with DSA, 16 detector row $\mathrm{CT}$ angiography is an accurate and reliable noninvasive method. In assessment of peripheral arterial disease in aortoiliac and lower limb arteries. ${ }^{[17]}$ In our study, MDCT was better than colour doppler ultrasound in detecting the infra popliteal the segment blocks and the length. In our study, out of 35 patients, 4 patients had history of trauma, one patient had complete occlusion of SFA. One patient had occlusion of ATA, one patient Femoro popliteal junction occlusion \& one patient had popliteal \& ATA occlusion, which was detected on both doppler \& MDCT. Fatihkantarci et al, indicated that in detecting arterial emergencies, doppler ultrasonography is a sensitive test, and it can be used as a substitute for screening arteriography. ${ }^{[18]}$ Rieger et al, found that MDCT had $99 \%$ sensitivity and $87 \%$ specificity in initial diagnostic technique to depict arterial injury with extremity trauma. ${ }^{[19]}$ The above studies indicate that both doppler \& MDCT have excellent sensitivity \& specificity in detecting lower limb arterial injuries, which is also shown in our study. MDCT has many advantages like the visualization of collaterals arising from the steno-occlusive segments of arteries in evaluating the lower limb arteries over color doppler. Previous studies have shown that doppler is capable of detecting the collaterals, one such study was done by Sharma et al, evaluated the presence of collaterals in peripheral arterial disease by color doppler. They found doppler was good in detecting the collaterals. ${ }^{[20]}$ In our study, the presence of collaterals were well shown by MDCT, however our study did not include the assessment of collaterals, but it is worthwhile to mention that MDCT is by far superior in detecting the collaterals than color doppler. The other most important advantage of MDCT over doppler is in assessing the length of steno-occlusive segments. MDCT will show the exact length of segment involved, which is very helpful in evaluation of status $\&$ treatment planning of peripheral arterial disease. Color doppler is also helpful in assessing the length of segments, bu, the disadvantage of doppler is that it is operator dependent $\&$ also sometimes the habitus of patient does not allow to assess the exact length of occluded segment. MDCT is superior in assessing the length of segments than color doppler. Ct angiography is less invasive, less expensive, and causes less radiation when compared with conventional catheter angiography and it also assesses aspects external to the lumen of the vessel that DSA cannot, including mural thrombus, atheroma, inflammation, and periarterial tissues. So MDCT \& doppler should be the initial modality of choice in assessing PVD, and DSA should be considered in only selected clinically difficult cases. The main competitor to MDCT is contrast-enhanced dynamic MRA. MRA has an advantage that it is unaffected by the presence of vascular calcification. However, MDCT scores over MRA by being much faster, less expensive and more widely available with better patient compliance. Leiner et al, found that contrastenhanced MR angiography is more sensitive and specific for diagnosis and pre interventional work-up of pad compared the diagnostic accuracies of color duplex ultrasonography (us). ${ }^{[21]}$ Ouwendijk et al. Found that interpretations of MR angiography and MDCT angiography for peripheral arterial disease have an excellent inter observer agreement. ${ }^{[22]}$

The above studies suggest that both MDCT \& MRA are equally good in evaluating the peripheral arterial disease.

\section{Conclusion}

In our study Doppler showed very good sensitivity, specificity, PPV, NPV and accuracy. Doppler - when compared to MDCT. Despite the limitations, it showed very good results in diagnosing complete occlusion, significant stenosis, and the patency of distal vessel. Thus color doppler is a non-invasive initial imaging modality of choice in suspected patients with PAD. MDCT has shown that it is accurate in identifying the degree, severity and level of peripheral vascular disease. It has excellent spatial resolution and provides route map thereby playing crucial role in decision making for management. The current generation CT angiography is a powerful tool for 
noninvasive imaging and treatment planning of pad. Our study shows that both color doppler \& MDCT can be complimentary in evaluation of lower limb arterial disease.

\section{References}

1. Hiatt WR, Goldstone J, Jr SCS, McDermott M, Moneta G, Oka R. Atherosclerotic Peripheral Vascular Disease Symposium Ii: Nomenclature For Vascular Diseases. Circulation. 2008;118(25):2826-2835. Available from: https://doi.org/10. 1161/circulationaha.108.191171.

2. Selvamani M, Yamunadevi A, Basandi PS, Madhushankari GS. Analysis of prevalence and clinical features of multicystic ameloblastoma and its histological subtypes in South Indian sample population: A retrospective study over 13 years. J Pharm Bioallied Sci. 2014;6(Suppl 1):S131-4. Available from: https://dx.doi.org/10.4103/0975-7406.137419.

3. Abdulhannan P, Da R. Homer-Vanniasinkam S. Peripheral Arterial Disease: A Literature Review. $\mathrm{Br}$ Med Bull. 2012;104(1):21-39.

4. Premlatha G, Subramaniam R, Deepa J. Prevalence And Risk Factors Of Peripheral Vascular Disease In A Selected South Indian Population. Diabetes Care. 2000;23(9):1295-300. Available from: https://doi.org/10.2337/diacare.23.9.1295.

5. Burrill J, Dabbagh Z, Gollub F, Hamady M. Multidetector computed tomographic angiography of the cardiovascular system. Postgrad Med J. 2007;83(985):698-704. Available from: https://dx.doi.org/10.1136/pgmj.2007.061804.

6. Parikh C, Brahmbhattp R. Efficacy Of Colour Doppler Imaging Over Ct Angiography In Peripheral Arterial Disease. Jintegr Health Sci. 2017;5(2):45-52.

7. Chidambaram P, Swaminathan RK, Ganeshan P. Doppler Ultrasound Can Be Used As A First Investigation Of Pad. J Clinton Diagn Res. 2016;10(2):12-16.

8. Shirol RJ, Shetty A, K CT. Role Of Mdet In Evaluation Of Peripheral Vascular Disease Of The Lower Limb Arteries And Comparison With Colour Doppler. J Evol Med Dent Sci. 2015;4(54):9336-9346. Available from: https://dx.doi.org/10. 14260/jemds/2015/1357.

9. Hasa R, Skana A. Mdet And Colour Doppler Used For Assessment Of Peripheral Vascular Disease Of Lower Extremities. Allan Med J. 2016;1:28-33.

10. Rv P, Vr R, Monthampally S. Mdct Angiogram V/S Colour Doppler Ultrasound In The Diagnosis Of Peripheral Arterial Diseases Of Lower Extremities. J Evolution Med Dent Sci. 2016;5(63):4457-4461.

11. Satyabhuvansinghnetam R, Singh. Ct Angiography Evaluation Of Pvd And Comparison With Colour Doppler. J Evol Med Dent Sci. 2015;14(83):14504-14514. Available from: https://www.researchgate.net/deref/http\%3A//dx. doi.org/10.14260/jemds/2015/2064.

12. Ota H, Takase K, Igarashi K, Chiba Y, Haga K, Saito H, et al. Mdct Compared With Digital Subtraction Angiography For Assessment Of Lower Extremity Arterial Occlusive Disease:
Importance Of Reviewing Cross Sectional Images. Am J Roentgenol. 2004;182(1):201-209. Available from: https://doi. org/10.2214/ajr.182.1.1820201.

13. Duddalwar VA. Multislice CT angiography: a practical guide to $\mathrm{CT}$ angiography in vascular imaging and intervention. Br J Radiol. 2004;77(1):27-38. Available from: https://doi.org/10. $1259 / \mathrm{bjr} / 25652856$.

14. Albrecht T, Foert E, Holtkamp R, Kirchin MA, Ribbe C, Wacker FK, et al. 16-MDCT angiography of aortoiliac and lower extremity arteries: comparison with digital subtraction angiography. Am J Roentgenol. 2007;189(3):702-711. Available from: https://doi.org/10.2214/ajr.07.2333.

15. Schertler T, Wildermuth S, Alkadhi H, Kruppa M, Marincek B, Boehm T. Sixteen-Detector Row CT Angiography for Lower-Leg Arterial Occlusive Disease: Analysis of Section Width. Radiology. 2005;237(2):649-656. Available from: https://dx.doi.org/10.1148/radiol.2372041861.

16. Kayhan A, Palabiyik F, Serinsoz S, Al E. Multidetector Ct Angiography Versus Arterial Duplex Usg In Diagnosis Of Mild Lower Extremity Peripheral Arterial Disease. Is Multidetector Ct A Valuable Screening Tool? Eur J Radiol. 2012;81(3):542548. Available from: https://doi.org/10.1016/j.ejrad.2011.01. 100.

17. Romano M, Mainenti PP, Imbriaco M, Amato B, Markabaoui $\mathrm{K}$, Tamburrini $\mathrm{O}$, et al. Multidetector Row Computed Tomographic Angiography Of Abdominal Aorta And Lower Limb Arteries. A New Diagnostic Tool In Patients With Paod. Eur J Radiol. 2004;50(3):303-308. Available from: https://doi. org/10.1016/s0720-048x(03)00118-9.

18. Kantarci F, Mihmanli I, Kara B, Bozlar U. Acute arterial emergencies: evaluation by Doppler ultrasound. Emerg Radiol. 2005;11(6):315-321. Available from: https://doi.org/10.1007/ s10140-005-0429-9.

19. Rieger M, Mallouhi A, Tauscher T, Lutz M, Jaschke WR. Traumatic Arterial Injuries of the Extremities: Initial Evaluation with MDCT Angiography. Am J Roentgenol. 2006;186(3):656-664. Available from: https://dx.doi.org/10. 2214/ajr.04.0756.

20. Sharma NC, Meena ML. Color duplex evaluation of peripheral arterial occlusive disease of lower extremities and to determine relationship between severity and number of collaterals associated. Indian J Radiol Imaging. 2005;15(4):587-587. Available from: https://dx.doi.org/10.4103/0971-3026.29180.

21. Leiner T, Alphons GH, Kessels PJ, Nelemans G, Boudewijn C, Vasbinder, et al. Peripheral arterial disease: comparison of color duplex US and contrast-enhanced MR angiography for diagnosis. Radiology. 2005;235:699-708. Available from: https://doi.org/10.1148/radiol.2352040089.

22. Ouwendijk R, Kock MCJM, Visser K, Pattynama PMT, de Haan MW, Hunink MGM. Interobserver Agreement For The Interpretation Of Contrast-Enhanced 3d Mr Angiography And Mdct Angiography In Peripheral Arterial Disease. Am J Roentgenol. 2005;185:1261-1267. Available from: https: //doi.org/10.2214/ajr.04.1296. 
Copyright: (C) the author(s), 2020. It is an open-access article distributed under the terms of the Creative Commons Attribution License (CC BY 4.0), which permits authors to retain ownership of the copyright for their content, and allow anyone to download, reuse, reprint, modify, distribute and/or copy the content as long as the original authors and source are cited.

How to cite this article: Prasad MBV, Boppana S, Reddy M, Juvvadi N, R, R. Computed Tomography Angiography Evaluation of Peripheral Arterial Disease and Correlation with Colour Doppler. Asian J. Med. Radiol. Res. 2020;8(2):73-79.

DOI: dx.doi.org/10.47009/ajmrr.2020.8.2.12

Source of Support: Nil, Conflict of Interest: None declared. 